\title{
Unión femoro tibio patelar en las especies domésticas
}

\author{
Bode, F.F.; Resoagli, E.H.; Cao, J.A.; Millán, S.G.; Resoagli, J.M.; Vargas Timoniuk, D. \\ Cátedra de Anatomía Comparada Primera Parte, Facultad de Ciencias Veterinarias, UNNE, \\ Sargento Cabral 2139, Corrientes (3400), Tel/fax 03783-425753. Email: anato1@vet.unne.edu.ar.
}

\begin{abstract}
Resumen
Bode, F.F.; Resoagli, E.H.; Cao, J.A.; Millán, S.G.; Resoagli, J.M.; Vargas Timoniuk, D.: Unión femoro tibio patelar en las especies domésticas. Rev. vet. 19: 2, 139-142, 2008. Se comunican las variaciones anatómicas encontradas en los medios de unión de la articulación femoro tibio patelar de las especies domésticas. Se procesaron articulaciones femoro tibio patelares de equinos $(n=10)$, grandes rumiantes $(n=6)$, pequeños rumiantes $(n=4)$, cerdos $(n=4)$ y carnívoros $(n=8)$, siguiendo las técnicas clásicas para la disección de modelos anatómicos. Las piezas fueron documentadas fotográficamente y conservadas en solución de Lawkosky modificada. Los hallazgos se comparan con las descripciones efectuadas para esta articulación por los autores clásicos de la anatomía comparada, constatándose la ausencia de información sobre algunas estructuras halladas en el presente trabajo. Tal es el caso de los ligamentos tibio meniscales detectados en caninos y el ligamento cruzado craneal encontrado en rumiantes, cerdos y carnívoros. Tales conocimientos aportan al acrecentamiento del acervo anatómico y pueden resultar de utilidad para la cirugía y traumatología veterinarias.
\end{abstract}

Palabras clave: animales domésticos, anatomía, unión femoro tibio patelar.

\begin{abstract}
Bode, F.F.; Resoagli, E.H.; Cao, J.A.; Millán, S.G.; Resoagli, J.M.; Vargas Timoniuk, D.: Femoro-tibio-patellar joint in domestic species. Rev. vet. 19: 2, 139-142, 2008. Anatomical variations found in femoro-tibio-patellar joint of domestic species, are discussed. Using classic techniques for the dissection of anatomical models, femoro-tibio-patellar joints of horses $(n=10)$, large ruminants $(n=6)$, small ruminants $(n=4)$, pigs $(n=4)$ and carnivorous $(\mathrm{n}=8)$, were processed. Pieces were documented photographically and conserved in Lawkosky modified solution. Findings were compared with the descriptions previously made by classic authors for this joint. We determine the lack of information regarding some anatomical structures, such as the tibio-meniscal ligaments found in dogs, and the cranial crossed ligament found in ruminants, pigs and carnivorous. This data may contribute to the knowledge of animal anatomy, and will probably be useful for surgery as well as veterinary traumatology.
\end{abstract}

Key words: domestic animals, anatomy, femoro-tibio-patellar joint.

\section{INTRODUCCIÓN}

Desde el punto de vista funcional, la unión de la babilla juega un rol importante tanto en la estática como en la dinámica del aparato locomotor de los mamíferos domésticos. En la unión femoro tibio patelar, las caras articulares son los cóndilos del fémur y los cóndilos de la tibia, entre los que se ubican los meniscos, que facilitan la coaptación de las superficies articulares. En la tibia los cóndilos convergen hacia su parte media formando la eminencia intercondílea, dividida por una depresión central, el área intercondílea central en los tubérculos, uno lateral y otro medial.

Recibido: 28 agosto 2008 / Aceptado: 8 octubre 2008
Hacia craneal de la eminencia intercondílea existen dos superficies rugosas destinadas a la inserción de los extremos dorsales de ambos meniscos. La tuberosidad tibial, situada en craneal de la extremidad, es de forma triangular a base proximal. Salvo en el equino, en que se encuentra dividida en dos tubérculos por una fosa intermedia, en el resto de las especies domésticas es indivisa.

En general, las partes que componen la unión femoro tibio patelar en los mamíferos son similares, no obstante, en las disecciones que habitualmente efectuamos con fines docentes y de investigación, en reiteradas ocasiones se observaron modificaciones en sus estructuras, particularmente en el nivel de inserción de los meniscos. 
En tal sentido, la mayoría de los autores consultados coincide en que las inserciones de las extremidades craneales de los meniscos se localizan en las fosas que limitan craneal, lateral y medialmente la espina tibial, mientras que la extremidad caudal varía según se trate del menisco lateral o medial. Algunos autores, al describir la inserción de los meniscos en el cerdo y el perro, mencionan la presencia de un ligamento transverso craneal que une los extremos de ambos meniscos ${ }^{2,7,8}$. Otros puntualizan la existencia de un ligamento transverso en carnívoros y -en algunos casos-en bovinos ${ }^{5}$. También se afirma que, en cerdos y perros, el ligamento transverso une los extremos craneales de ambos meniscos ${ }^{3}$.

Un autor describe el entrecruzamiento de las inserciones craneales de ambos meniscos en bovinos, mencionando además la presencia de una bandeleta fibrosa transversal que une los extremos craneales de ambos meniscos en carnívoros y conejos, pero que no existe en el resto de las especies domésticas ${ }^{6}$. También se ha descrito una rama tibial caudal supernumeraria en el menisco medial del equino, que se inserta en la incisura poplítea ${ }^{1}$. Otros autores mencionan que en los carnívoros el ligamento transverso une ambos extremos de los meniscos y la tibia ${ }^{4}$.

El presente trabajo, parte del Programa de Revisiones Anatómicas realizado por la Cátedra Anatomía Comparada Primera Parte, tuvo como objetivo corroborar la existencia de las variaciones en la inserción de los meniscos de la articulación femoro tibio patelar en las distintas especies de interés veterinario.

\section{MATERIAL Y MÉTODOS}

Fueron estudiadas diez uniones femoro tibio patelares de equinos, seis de grandes rumiantes, cuatro de pequeños rumiantes, cuatro de cerdos y ocho de carnívoros, a partir de disecciones realizadas según las técnicas clásicas para la obtención de modelos anatómicos. Los hallazgos fueron documentadas fotográficamente y las piezas fueron conservadas en solución de Lawkosky modificada.

\section{RESULTADOS Y DISCUSIÓN}

El estudio de las disecciones efectuadas permite corroborar un patrón anatómico común en la estructura general de la babilla de los mamíferos domésticos, pero también variaciones relacionadas a la disposición, trayecto e inserción de los ligamentos que sujetan los meniscos y al comportamiento del ligamento cruzado craneal.

En un equino se observó la presencia de un ligamento menisco caudal medial supernumerario, extendido desde el borde abaxial del menisco medial a la incisura poplítea de la tibia (Figura 1).

En rumiantes, el mayor espesor del menisco lateral fue constante. En el $90 \%$ de los casos la inserción de los extremos craneales de ambos meniscos se entrecruzaron de tal manera que el menisco medial lo hizo en el ángulo lateral de la base de la tuberosidad tibial y la extremidad craneal del menisco lateral lo hizo en el ángulo medial (Figuras 2 y 3).

En cerdos, los extremos craneales de los meniscos se insertaron de manera similar a los de los rumiantes. Además, estuvieron unidos entre sí a través de un ligamento transverso craneal (Figura 4).

El ligamento cruzado craneal de rumiantes, cerdos y carnívoros se diferenció de su homólogo del equino porque en aquéllos, se dividió en dos ramas, una craneal y superficial que se insertó entre los extremos craneales de los meniscos y otra caudal que lo hizo en el área intercondílea central (Figuras 2, 3 y 4).

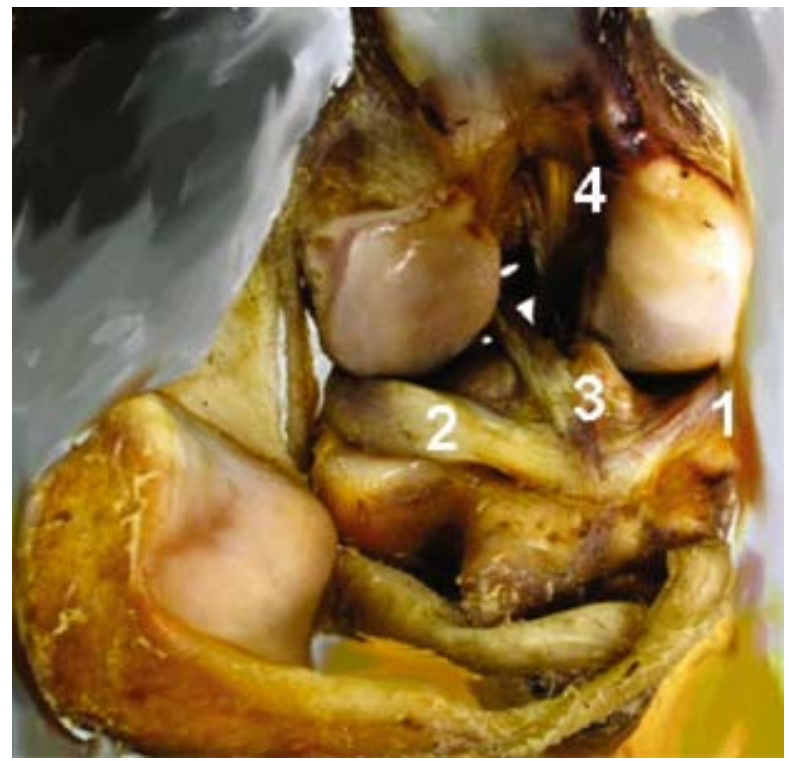

Figura 1. Babilla del equino, vista craneal. 1: inserción craneal del menisco medial, 2: inserción craneal del menisco lateral, 3: inserción del ligamento cruzado anterior, 4: origen del ligamento cruzado posterior.

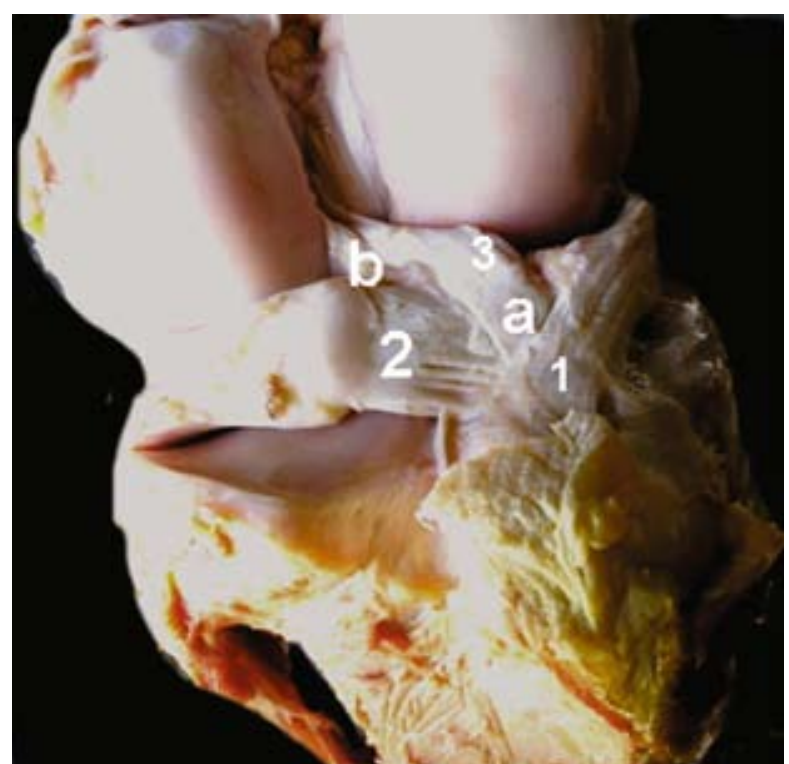

Figura 2. Babilla de grandes rumiantes, vista craneal. 1: inserción craneal del menisco medial, 2: inserción craneal del menisco lateral, 3: ligamento cruzado anterior (a: rama superficial, b: rama profunda). 
En carnívoros, la inserción del extremo craneal de los meniscos y la presencia del ligamento transverso respondieron a las descripciones clásicas (Figura 5). En la membrana fibrosa de la cápsula articular de los carnívoros se observaron espesamientos situados a ambos lados del borde abaxial de los meniscos, conformando ligamentos que se prolongaron hasta los costados de los cóndilos de la tibia, a los que se denominó ligamentos tibiomeniscales lateral y medial respectivamente (Figura 6).

Los hallazgos efectuados en el presente trabajo referentes al entrecruzamiento de las inserciones del extremo craneal de ambos meniscos en grandes rumiantes, coinciden con los reportados por otro autor ${ }^{6}$. Por el contrario, la división en dos ramas del ligamento cruzado craneal no es mencionada en dicha obra. La

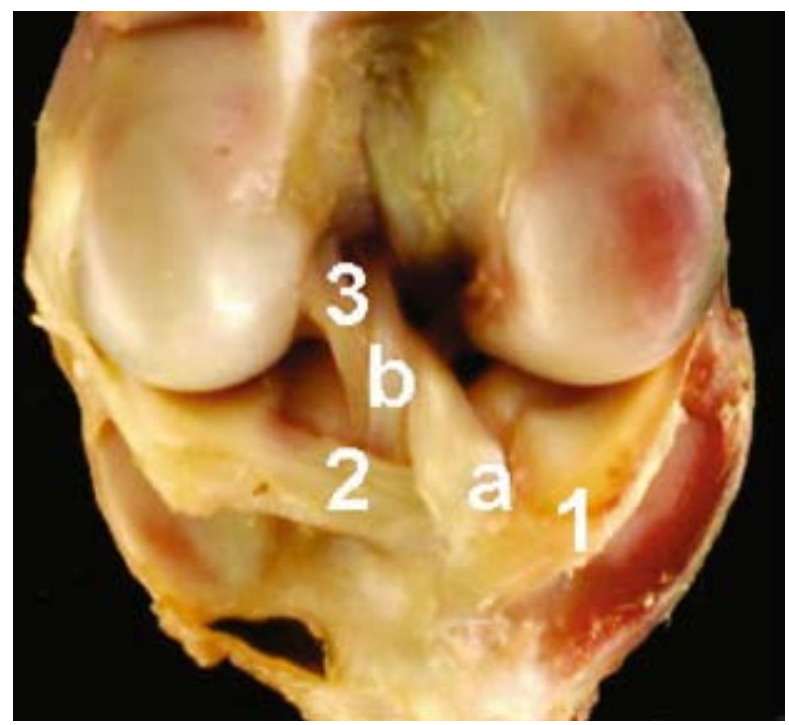

Figura 3. Babilla de pequeños rumiantes, vista craneal. 1: inserción craneal del menisco medial, 2: inserción del menisco lateral, 3: ligamento cruzado craneal (a: rama superficial, b: rama profunda).

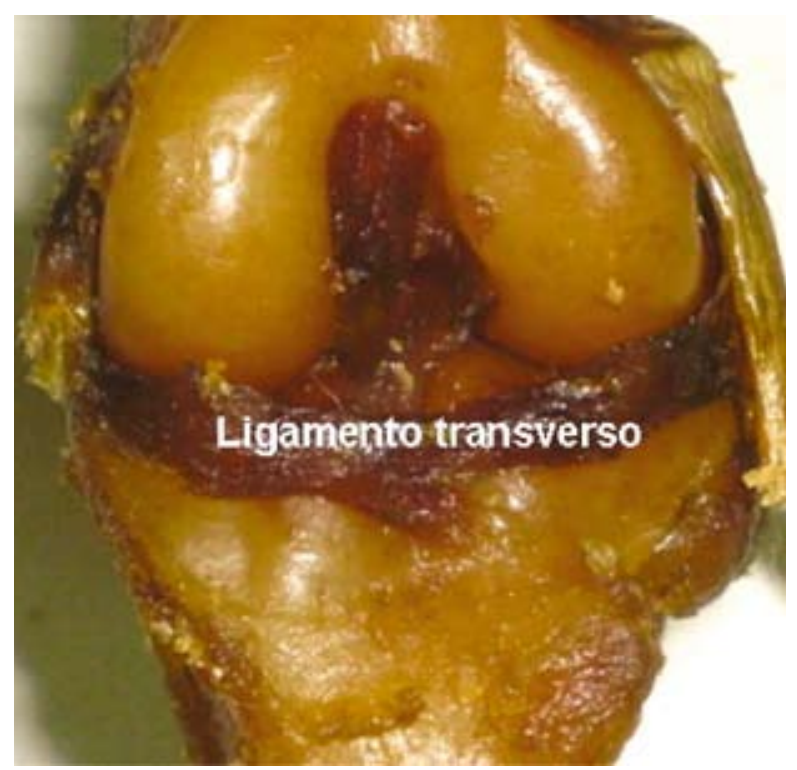

Figura 5. Babilla del perro, vista craneal con exposición del ligamento transverso. presencia del ligamento transverso craneal en perros y cerdos es concordante con las descripciones efectuadas por varios autores ${ }^{2,7,8}$. La bibliografía consultada no consigna la presencia de los ligamentos tibio meniscales observados en nuestras disecciones en caninos, como así tampoco la del ligamento cruzado craneal de rumiantes, cerdos y carnívoros.

\section{REFERENCIAS}

1. Bode FF, Resoagli EH, Millán SG. 1996. Ligamento tibial caudal menisco medial supernumerario en equinos. Vet $\operatorname{Arg}$ 12: 38-40.

2. Dyce KM, Sack WO, Wensing CJ. 1991. Anatomía Veterinaria, Panamericana, México, p. 98-100.

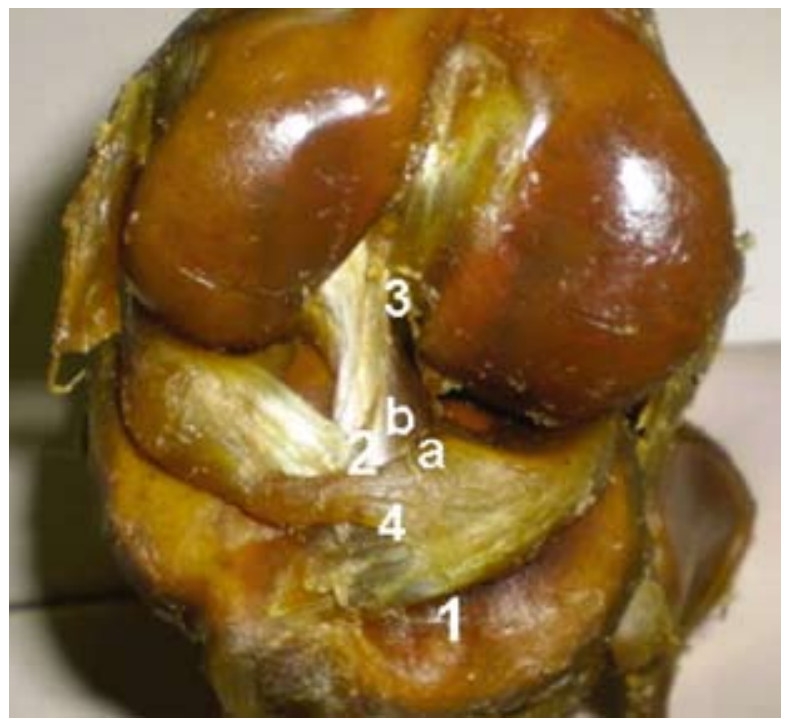

Figura 4. Babilla del cerdo, vista craneal. 1: inserción craneal del menisco medial, 2: inserción del menisco lateral, 3: ligamento cruzado craneal (a: rama superficial, b: rama profunda), 4: ligamento transverso.

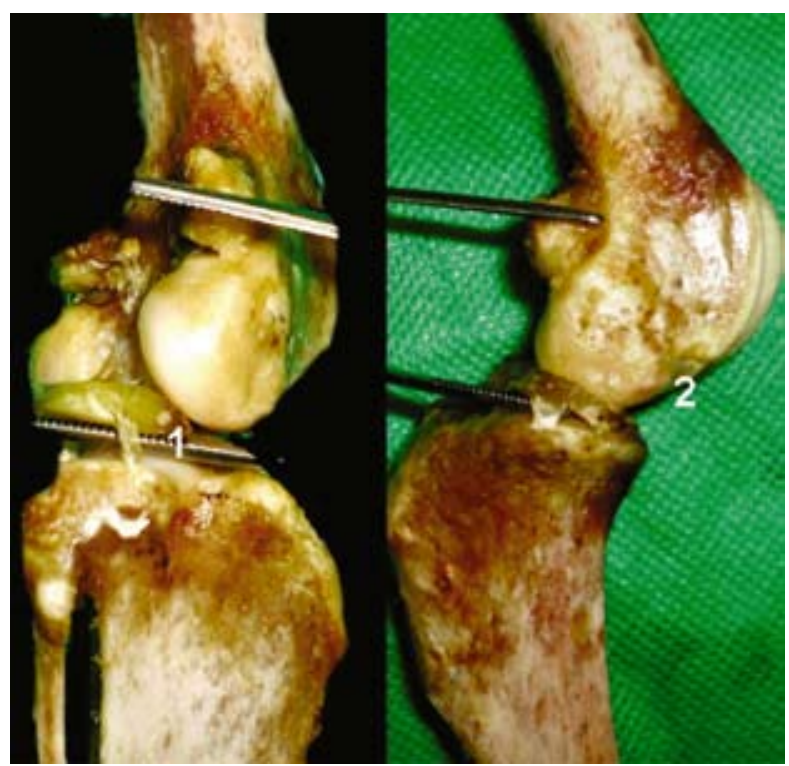

Figura 6. Babilla del perro. Ligamento tibio meniscal (1: lateral, 2: medial). 
3. Getty R.1982. Anatomía de los Animales Domésticos, $5^{\circ}$ ed., Salvat, Barcelona, p. 414-416, 876, 1382, 1651.

4. Gimeno M, Gil García F, Laborda VJ. 1998. Anatomía del Perro. Protocolos de Disección, $2^{\mathrm{a}}$ ed., Masson, Zaragoza, p. 203-210.

5. König E, Liebich HG. 2005. Anatomía de los Animales Domésticos, Panamericana, Barcelona, p. 205, 215, 227 230.
6. Martínez Quiroga, C. 1958. Anatomía Comparada, Ed. FUNE, Corrientes, $72 \mathrm{p}$.

7. Schwarze E. 1972. Compendio de Anatomía Veterinaria, Acribia, Zaragoza, p. 154-157.

8. Sisson S, Grossman JD. 1963. Anatomía de los Animales Domésticos, $4^{\circ}$ ed., Salvat, Barcelona, p. 240-246. 